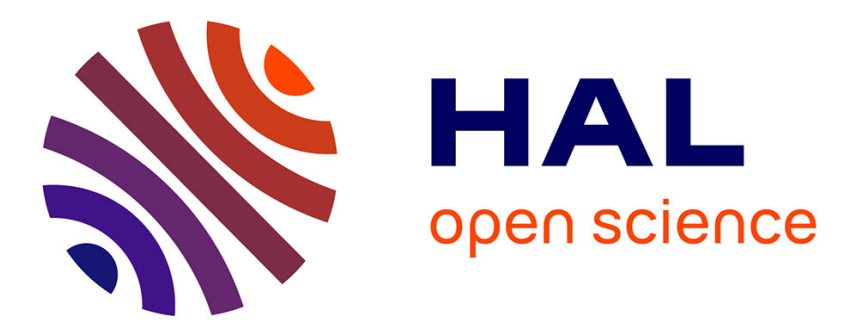

\title{
Longitudinal fuzzy control for autonomous overtaking
} Joshué Pérez Rastelli, Vicente Milanés, Enrique Onieva, Jorge Godoy, Javier Alonso

\section{To cite this version:}

Joshué Pérez Rastelli, Vicente Milanés, Enrique Onieva, Jorge Godoy, Javier Alonso. Longitudinal fuzzy control for autonomous overtaking. Proceedings of the 2011 IEEE International Conference on Mechatronics, Apr 2011, Istanbul, Turkey. hal-00737465

\section{HAL Id: hal-00737465 \\ https://hal.inria.fr/hal-00737465}

Submitted on 1 Oct 2012

HAL is a multi-disciplinary open access archive for the deposit and dissemination of scientific research documents, whether they are published or not. The documents may come from teaching and research institutions in France or abroad, or from public or private research centers.
L'archive ouverte pluridisciplinaire HAL, est destinée au dépôt et à la diffusion de documents scientifiques de niveau recherche, publiés ou non, émanant des établissements d'enseignement et de recherche français ou étrangers, des laboratoires publics ou privés. 


\title{
Longitudinal fuzzy control for autonomous overtaking
}

\author{
Joshué Pérez, Vicente Milanés, Enrique Onieva, Jorge Godoy and Javier Alonso \\ Centre for Automation and Robotics (UPM-CSIC), Madrid, Spain \\ (e-mail: joshue.perez, vicente.milanes, enrique.onieva, jorge.godoy, javier.alonso)@car.upm-csic.es)
}

\begin{abstract}
Cooperative maneuver among autonomous and conventional vehicles can be considered one of the next steps for obtaining a safer and more comfortable driving. Some examples of these maneuvers are: Adaptive Cruise Control (ACC), intelligent intersection management or automatic overtaking maneuvering, among others. One of the most important aims of the Intelligent Transportation Systems (ITS) is to use roads without substantial modifications of the current infrastructure. The overtaking maneuver demands special attentions, in particular when it is carried out on two-way roads. For this reason, a fuzzy decision system based on fuzzy logic able to execute an autonomous overtaking in two-way roads has been implemented in this paper. The experiments are focused on the longitudinal control when an autonomous vehicle is overtaking to other. Moreover, different cases have been considered using an oncoming vehicle from the other direction on a two-way road. Several trials with three real vehicles communicated via a wireless network were carried out, showing a good behavior.
\end{abstract}

Index Terms-Intelligent Transportation Systems, Fuzzy Logic, Overtaking Manuver.

\section{INTRODUCTION}

In the last decade, significant advances on research and commercial purposes focused on vehicular applications have permitted to improve the safety in both urban and highway roads. Moreover, vehicles capable of driving in controlled urban environments during several hours have been developed [1]. However, fully autonomous vehicles capable of driving along real roads by themselves - that is, coexisting with conventional vehicles - are still an utopian target.

The implementation of cooperative maneuver requires the availability in real time of information about vehicles driving in the same area - i.e., a vehicle coming into a roundabout needs to know the information about which vehicles are presently driving in the roundabout. Of all of cooperative maneuver, overtaking in a two-way road is one of the most risky, because the action to be taken depends on a great quantity of factors such as: distance to bends or intersections, positions and speed of the surrounding vehicles, etc.

This work is focused on the analysis and tests of overtaking maneuver, since they have not received too much attention and constitute one of the less studied cooperative maneuver in the field of autonomous vehicles. Our approach is based on control actions over the overtaking vehicle, maintaining the same infrastructure, since, for example, in Spain the $91.7 \%$ of the roads are still simple two-way roads, and only the rest (an $8.3 \%$ have two or more lanes in the same direction [2]. The autonomous lateral control for lane-change maneuvers have been previously developed [3], so the present work is focused in the longitudinal control in case of a vehicle is overtaking to other, and other vehicle oncoming from the front.

The rest of the paper is organized as follows. In section II, an overview of the related works in the autonomous overtaking maneuver is described. The description of the test environment and vehicles used is shown in section III. Section IV details the decision control algorithms based on fuzzy logic, and section V shows the experiments that validated our approach. Finally some remarks and conclusion are included in section VI.

\section{RELATED WORKS}

The lane-change maneuver is the base of an overtaking. In this topic, the California Partners for Advanced Transit and Highways (PATH) program has presented the most relevant advances in the last few years. Jula [4] defined the different phases for a safe lane-change. Other studies and demonstrations of the PATH have been carried out in [5], considering platooning with several real vehicles permitting lane-change maneuvers. Furthermore, fuzzy controller applied to the steering wheel [6] has been developed in the last years on real autonomous vehicles.

Other authors have simulated the overtaking using a multiple-goal reinforcement learning framework [7] showing the overtaking maneuver in different situations, but it has only been tested in simulations. Most recently, the University of Ulster, in Northern Ireland, have been working in microscopic model (behavior of individual vehicles), which considerate the actions of the close cars [8]. Finally, other simulations considering trajectory-generation method for autonomous overtaking capable of handling emergency situations and aborting the maneuver have been developed in [9].

Finally, it is noteworthy in this field the Defense Advanced Research Projects Agency (DARPA) Urban Challenge, since is one of the most important world wide meetings in autonomous vehicles [1]. This competition is organized by the U.S. Government, and its aim is to achieve an autonomous driving with real vehicles in urban environments. Junior, the participant of the Stanford University, won the second place in the last edition and was capable of negotiating intersections and performing lane-changes[10].

The AUTOPIA program, where this work has been realized, at the Center for Automation and Robotics (CAR), UPM CSIC (Universidad Politécnica de Madrid - Consejo Superior de Investigaciones Científicas), Spain) has been worked during 
last 15 years in the development of vehicles capable of driving autonomously. Different algorithms and strategies have been tested in simulation [11]. Moreover, maneuver with real vehicles, changing as less as possible both the environment and the vehicles [3] have been done. These vehicles use a real time kinematic-differential global positioning system (RTKDGPS) to acquire the position with a precision up to two centimeters. Currently, there are three real vehicles: two of them fully automated and other one driven manually; all of them are equipped with a DGPS and wireless communication to transmit its position and speed.

\section{TECHNICAL APPROACH}

In this section, the conditions of the longitudinal fuzzy controller for the overtaking maneuver are explained; taking into consideration the test environment, communication system and the basic concept used so far in the overtaking is shown below.

\section{A. Test environment}

AUTOPIA program has instrumented three vehicles: one Citroën Berlingo van and two Citroën C3, one of them is a convertible Pluriel. In the experiments only the overtaker vehicle (the Berlingo van) has been driven in autonomous mode, the other vehicles have been used for sending the information to be considered in the proposed control scheme. The overtaken (vehicle in front) and the vehicle that comes in the other lane will be driven manually.

The van is electrically powered. Its control architecture is shown in Figure 1. The main sensor to acquire environmental information is an RTK-DGPS. To automate the steering wheel, a computer controlled motor geared to the steering bar has been added. The accelerator generates a signal between 0 to 5 volts, this signal (in automatic mode) is mimicked by the computer using an I/O card which also measures the speed by looking at the pulse train from the car tachometer.

Two on board PCs have been used. This architecture has two separate levels of control: (i) one for receiving the signal from the GPS, WiFi card and the tachometer card - the main PC; (ii) the other hosts the guidance system, the knowledge base and the fuzzy control, and it is communicated with the other PC through an Ethernet connection [12].

Another PC is used for controlling the low level control (DC motors). These motors control the steering wheel and the brake. A new generation motor controller for the steering wheel is installed. This system includes a discrete PID, which permits to smooth the motor output. An incremental encoder to close the inner loop control is used. The targets are sent from the main computer. The control paradigm of the vehicles is divided in two basic controllers: lateral and longitudinal. Details about the lateral controller can be found in [3], [13]

The other vehicles have been driven in manual mode [12], and these are equipped with a DGPS and a laptop PC. A control program for sending data and checking the communications among the vehicles has been installed in the manually driven cars.

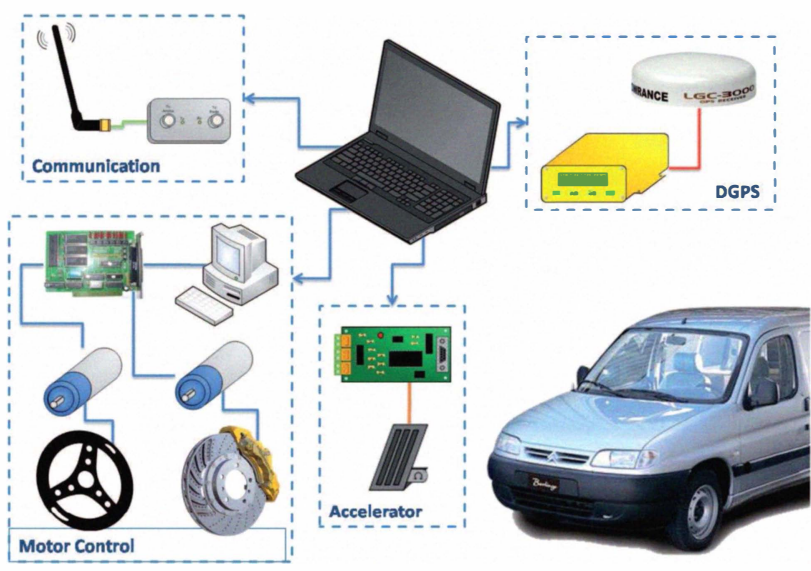

Fig. 1. Control scheme for the overtaker vehicle.

CAR's facilities emulate an urban circuit. The largest street has a total length of 220 meters with width of 6 meters. Because this length, the speed that it can be used in the experiments is limited at $30 \mathrm{~km} / \mathrm{h}$.

\section{B. Communication system}

The vehicle that is doing the overtaking needs to know the position and speed of the rest of vehicles in real time. In our system the DGPS is used as a real time clock for controlling and sending data with frequency of $5 \mathrm{~Hz}$.

For the communication, a wireless network connection among the vehicles is used. Each on board PC has a Proxim Orinoco $11 \mathrm{~b} / \mathrm{g}$ gold card, based in the $802.11 \mathrm{~b} / \mathrm{g}$ standard. The speed rate is $11 \mathrm{Mb} / \mathrm{s}$. A frame to exchange data among the cars is defined as follow: Name, UTM North position, UTM South position, real speed and possible alarm warnings. The package is less that 100 bytes, therefore the information is always available for the controlled vehicle into a range of 500 meters. All this information is used as inputs to the fuzzy controller in the overtaking maneuver.

\section{Overtaking maneuver}

An overtaking controller, based on expert knowledge has already been developed in a previous work [13]. But, so far, only two vehicles on a one-way road have been considered.

The classical overtaking maneuver consists on passing a vehicle or an obstacle that is in the predefined trajectory (in our case right-hand lane). The overtaking is defined as two consecutive lane changes, one from the right to the left lane, and other from the left to the right lane, once overpassed the overtaken vehicle.

There are two condition that must be considered to implement the overtaking in autonomous vehicles: one of them focuses in the interaction with the vehicle ahead, and the other with the environment. The requirements to carry out an overtaking are:

- The overtaken vehicle speed is lower than that of the overtaker vehicle. 


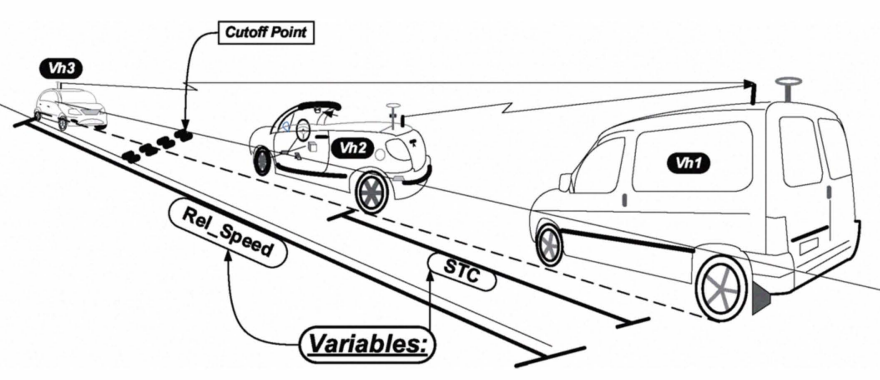

Fig. 2. Overtaking involving three vehicles on a two-way road.

- The overtaken vehicle is in the same lane as the overtaking one.

The external conditions are:

- The vehicles are moving in a straight road.

- The distance to a bend is enough to finish the maneuver completely.

- The left lane is free.

The sequence execution of the overtaking is as follow: If a vehicle is driving in front of the automatic one, the adaptive cruise control (ACC) system is activated. Once this occurs, the conditions to allow the overtaking maneuver are evaluated [13].

The overtaking can be separated in three phases [13]:

- Phase 1 is when the vehicle begins the maneuver; it is the first lane change from the right lane to the left lane. Before the aforementioned conditions are evaluated it is necessary to determinate if the distance is enough to allow the lane change [3].

- Phase 2 covers from the overtaker vehicle is in the left lane until the overtaken vehicle is passed.

- Phase 3 is used to define the return to the reference lane - the right lane.

\section{OVERTAKING WITH THREE VEHICLES}

The overtaking described in the previous section only involves two vehicles, that is, external conditions are always true. The next step, and the aim of this paper, is to consider other vehicle coming on the opposite way. Several dangerous situations are possible, e.g.: the overtaken vehicle increases suddenly its speed or there is a vehicle coming from the other lane. Figure 2 shows an overview about an overtaking involving three vehicles in a two-way road. In the same figure, $V h_{1}$ is the overtaken vehicle, $V h_{2}$ is the vehicle in front and $V h_{3}$ is the vehicle that comes on the opposite way.

In that situation a more detailed study is required. First, it is necessary to know when and how to stop the overtaking maneuver. For this reason, a fuzzy based algorithm has been developed, taking into account the expected human behavior in this kind of situations.

Safety is the imperative criterion in the design, so $V h_{1}$ must have enough time to return to the right lane, no matter the phase it might be. To this end, a Cutoff Point $\left(C_{P}\right)$ is defined as shown, graphically in figure 2 . This point is used as a dynamic

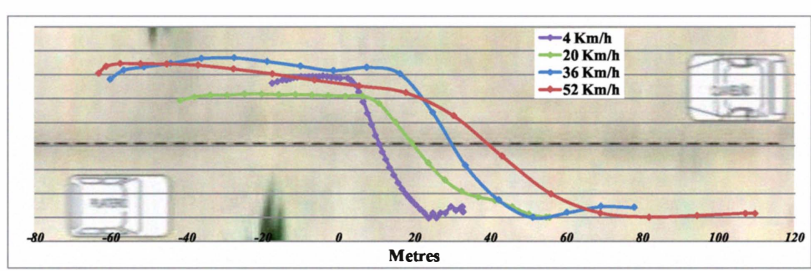

Fig. 3. Distance to complete the overtaking maneuver

referent for $V h_{1}$ and $V h_{3} . C_{P}$ always will be in front of $V h_{2}$, and is calculated in function of the speeds of $V h_{1}$ and $V h_{2}$. This point is used to determinate the time needed for $V h_{1}$ and $V h_{3}$ to arrive to a safety point and, in function of that, abort or not the overtaking.

To adjust the speed of the vehicle, it is necessary to know the time that $V h_{1}$ needs to perform a lane-change. In this sense, a study in function of the van speed of the lane change respects to the time has been done. Figure 3 shows the trajectories at the different speeds. These tests were carried out with the $V h_{2}$ stopped. A least squares solution of second order is used. This function is only valid for our vehicle with speeds between 4 and $52 \mathrm{~km} / \mathrm{h}$. The data has been collected with the overtaker vehicle $\left(V h_{1}\right)$ in autonomous mode.

Equation 1 shows the Total Distance $\left(T_{D}\right)$ in front of the vehicle $V h_{2}$, where the cutoff point is defined in function of $V h_{2}$ and $V h_{2}$ speeds ( $S_{1}$ and $S_{2}$ respectively) [3]. $L$ is the length of the vehicle ( $L=4.1$ meter $s)$.

$$
T_{D}=\left(0.014 V_{1}^{2}+0.2709 V_{1}+23.011\right)\left(1+\left(\frac{V_{2}}{V_{1}}\right)\right)+L
$$

The overtaking will be possible if $V h_{1}$ arrives to the $C_{P}$ before that $V h_{3}$. Now, It is important to set the time that each vehicle delay arriving to the $C_{P}$. Equations 2 and 3 show the calculations of these times.

$$
\begin{aligned}
& T v h_{1}=\frac{C_{P}-P_{1}}{V_{1}-V_{2}} \\
& T v h_{3}=\frac{P_{3}-C_{P}}{V_{1}+V_{3}}
\end{aligned}
$$

Where $C_{P}$ is the Cutoff Point position. $P_{1}$ and $P_{3}$ are the position of $V h_{1}$ and $V h_{3}$ respectively ( see Fig. 2). The $T v h_{1}$ is the time that $V h_{1}$ has to reach the $C_{P}$, it means, the time that $V h_{1}$ has to do a safe overtaking. The $T v h_{3}$ is the time that the $V h_{3}$ needs for arriving to the $C_{P}$ point. The overtaking is aborted when $T v h_{1}$ is greater than $T v h_{3}$ (dangerous situation).

The next section presents the longitudinal controller used on the phase 2 (when the vehicle is on the right lane) of the overtaking maneuver.

\section{LONGITUDINAL FUZZY CONTROL}

Based on the information that comes from other drivers, it is possible to obtain all the fuzzy control parameters. Emulating human driving, Zadeh [14] has proposed several examples where fuzzy control could be applied. Among them, 


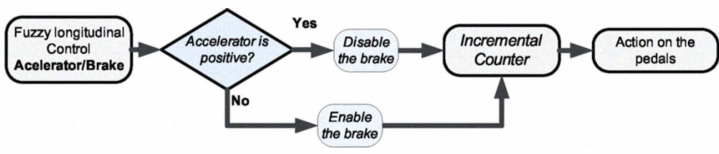

Fig. 4. Structure of longitudinal control

autonomous driving is an interesting field to apply fuzzy logic using human driver experience as expert knowledge.

AUTOPIA program has a wide experience in applications of fuzzy control in driverless vehicle applications [6], [15], based on ORBEX (Spanish acronym for experimental fuzzy coprocessor) [16]. ORBEX allows to write fuzzy sentences in an almost natural language. Simple rules like IF... THEN... are used; it allows defining variables and their fuzzy-rules and to join others variables in rules to get out crisp signals to controllers.

\section{A. Target following control}

For the longitudinal control, two variables are used: Speed error and Acceleration. They are calculated from the real speed of the vehicle, taken from the tachometer. To simplify the defuzzification process the input variables have triangular and trapezoidal shapes and the outputs are singletons, as Sugeno suggested in [17]. Simple syntax for the fuzzy rules are used [18], for instance:

IF Speed error MORE THAN null THEN Accelerator up IF Acceleration LESS THAN null THEN Accelerator down

Center of mass method implements the defuzzification operation [17]. The output is sent to the acceleration card.

Figure 4 shows the structure of the longitudinal control implemented. The fuzzy controller sends the output to an incremental counter, which is in charge of keep the throttle pedal pushed or not. A filtering stage orders to enable or disable the brake regarding the output signal of the fuzzy control. In this scheme the throttle has priority compared with the brake, like a human driver does, since less pushing on the throttle also means to brake.

The target following control, implemented for the longitudinal actuators, is always working when a normal overtaking (without any external conditions false) is running. However, if $T v h_{3}$ is more that $T v h_{1}$, it means, that the external conditions change adversely, then a extend controller is activated.

\section{B. Abort Fuzzy Controller for the overtaking}

The Abort Fuzzy controller (AFC) is activated when a collision between the overtaker vehicle $\left(V h_{1}\right)$ and the vehicle coming in the left lane $\left(V h_{3}\right)$ can occur. This controller reduces or increases the speed of the vehicle in function of the behavior of the involved vehicles and the environment conditions. Once the vehicles return to the right lane (finishing or intemupting the overtaking maneuver) the target following control is again activated.

Two new input variables have been defined. These are the Relative Speed (RelSpeed) and the Security Time for Collision
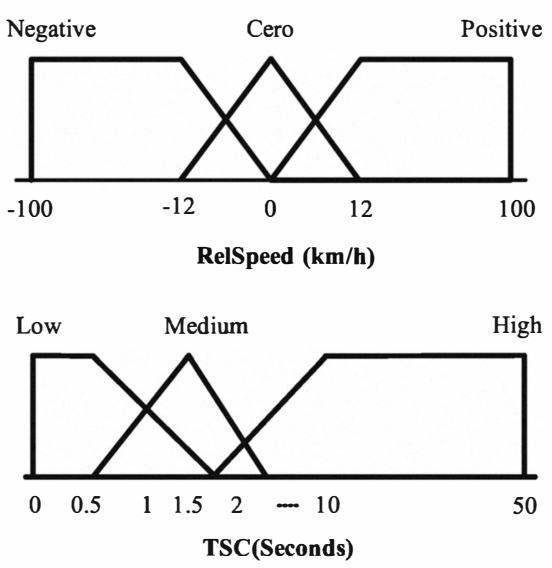

Fig. 5. Membership function of the AFC

\begin{tabular}{|c|c|c|c|}
\hline \multicolumn{5}{|c|}{ Throttle } \\
\hline STC/RelSpeed & Low & Medium & High \\
\hline Negative & lift & lift & tread \\
\hline Cero & lift & nothing & nothing \\
\hline Positive & lift & lift & lift \\
\hline \hline \multicolumn{4}{|c|}{ Brake } \\
\hline STC/RelSpeed & Low & Medium & High \\
\hline Negative & lift & nothing & lift \\
\hline Cero & nothing & lift & lift \\
\hline Positive & tread & lift & lift \\
\hline
\end{tabular}

TABLE I

FUZZY RULE FOR THE THROTTLE (TOP) AND BRAKE (BOTTOM) CONTROL

(STC). RelSpeed represents the difference between the speeds of $V h_{1}$ and $V h_{2}$ respectively, in $\mathrm{km} / \mathrm{h}$. In a normal overtaking its value is always positive, but when $V h_{2}$ increases its speed, RelSpeed tends to be negative. STC is the remaining time until $V h_{1}$ and $V h_{3}$ reaches the same position, in seconds; it is calculated as follows:

$$
T v h_{3}=\frac{\left(P_{3}-P_{1}\right) 3.6}{S_{1}+S_{3}}
$$

Where $P_{1}, P_{3}, S_{1}$ and $S_{3}$ are the positions and speeds of the $V h_{1}$ and $V h_{3}$ respectively. The positions are in meters and the speed in $\mathrm{m} / \mathrm{s}$. $S T C$ is always positive. The membership function of the variables RelSpeed and STC are shown in figure 5. According with experimental knowledge coming from expert driver, the values of this function has been experimentally tuned. The output variable is codified by means of three Sugeno's singletons [17]:

1) Lift (at -1 ; it means lift the maximum pedal)

2) Tread (at 1 ; it means push the maximum pedal)

3) Nothing (at 0 ; it means to keep the pedal pressure)

Singletons' values outputs are the same both the throttle as for the brake. Table I shows the fuzzy rules for the throttle and the brake pedals respectively.

The designed controller gives priority to the throttle respect to the brake. As was previously stated in Figure 4, the throttle and brake have never pushed at the same time. 


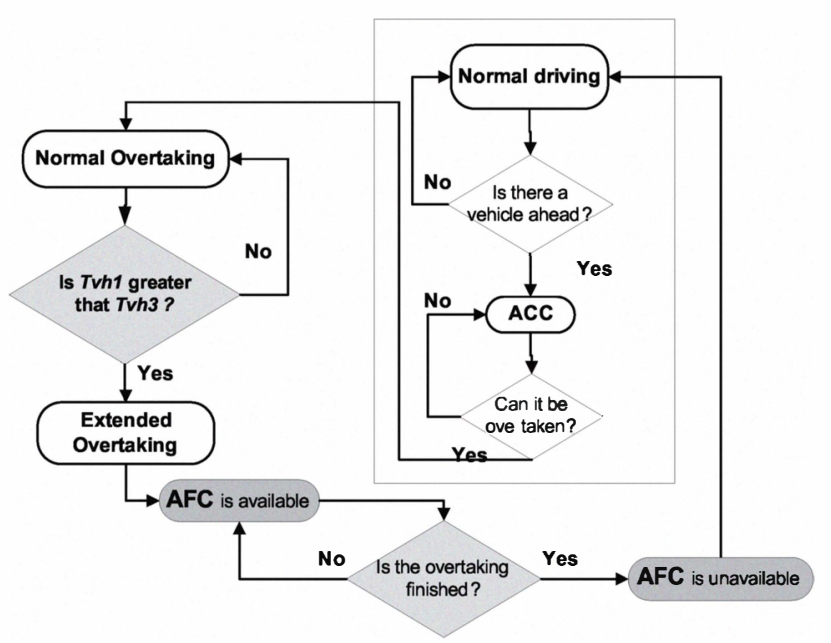

Fig. 6. Control architecture for autonomous driving

\section{Control architecture for aborting the overtaking}

In this section, a central control architecture for autonomous vehicles is presented. Figure 6 describes the different subcontrollers implemented in our vehicles. The decision system is sequential and structured. The conditions for each autonomous driving modes are explain as follows:

Normal driving is the first driving mode. It is when the vehicle just makes a line tracking. If the vehicle detects on the same lane other vehicle our controller changes to the $A C C$ mode, the second driving mode. Here, if the overtaking conditions are fulfilled, the vehicle starts a Normal overtaking. Finally, if it is necessary to abort the overtaking $\left(T v h_{1}>\right.$ $T v h_{3}$ ), then the vehicle in extend overtaking mode, and the AFC controller is activated.

The $A F C$ reduces the speed and returns to the right lane when the vehicle is far way to complete the maneuver. However, when the vehicle is completely in the left lane (in phase 2), the AFC can evaluate the condition, and if there is enough time (STC) and the RelSpeed is negative, it pushes the throttle and finishes quickly the overtaking. The $A F C$ is always inactivated when the overtaker vehicle is completely on the right lane.

\section{EXPERIMENTS WITH THE LONGITUDINAL OVERTAKING CONTROLLER}

The designed control system has been tested with three real vehicles in our private facilities. The experiments show good behavior in two operation modes: Normal overtaking and Extended overtaking (AFC controller). Only the overtaken vehicle $\left(V h_{1}\right)$ is unmanned, the others are manually driven. In the graphics we show the speed of the overtaken vehicle and the output of the longitudinal fuzzy control. Moreover, a sequence of pictures with a good performance of the maneuver in two different cases has been included.

The first experiment shows the result when the overtaken vehicle aborts the overtaking a few minutes of starting. Figure 7 shows the speed on the top part, and the fuzzy output
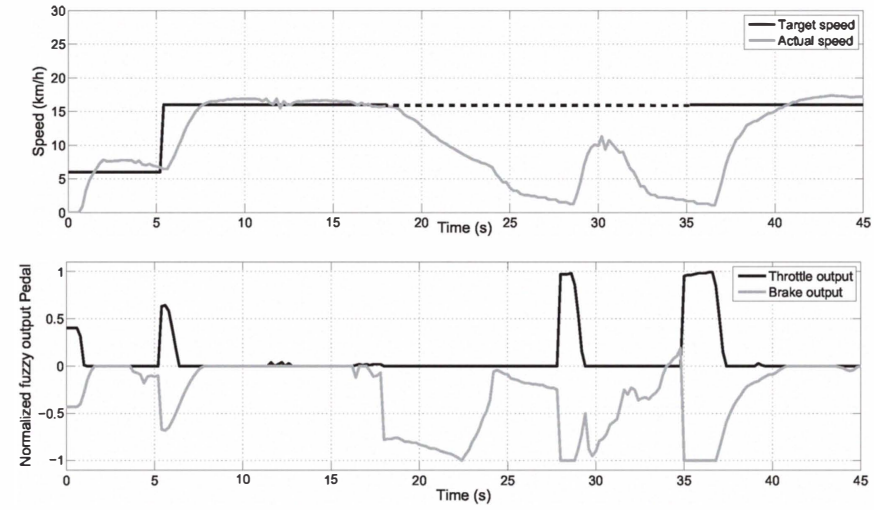

Fig. 7. Vehicle speed and fuzzy control outputs in the experiment 1

on the bottom. The gray line is the target speed, which is $6 \mathrm{~km} / \mathrm{h}$ at the beginning and $16 \mathrm{~km} / \mathrm{k}$ when the overtaking is running. The target following control maintains the speed while the overtaking. But, a sudden change in the external conditions make that $T v h_{1}$ is greater that $T v h_{3}$, and then the AFC is activated around 18 seconds (see Fig. 7). Dashed line represents that AFC has been activated. When the vehicle has returned to the right lane, then the target following control is again activated (around 35 second figure 7). Figure 9 shows the steps in different moments of this maneuver: the first picture shows when the vehicle is on the right lane with $V h_{1}$ ahead. The second is starting the lane-change, and the next one is with the vehicle almost on the left lane. The last picture shows that vehicle return to the right lane behind of $V h_{2}$.
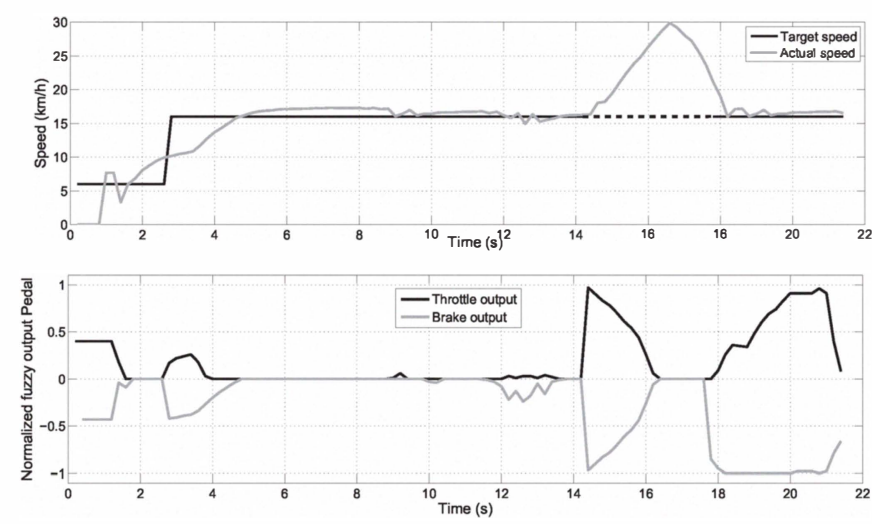

Fig. 8. Vehicle speed and fuzzy control outputs in the experiment 2

The second experiment shows the overtaken vehicle behavior when the condition permits to finish successful the overtaking maneuver. The controller increases the speed and then the vehicle returns safety to the right lane. Figure 8 shows the speed increasing at the around 15 second until $30 \mathrm{~km} / \mathrm{h}$. In this case, the vehicle that comes from the opposite way is far away, also the speed of the overtaker vehicle is lower than the first experiment. The evolution of this maneuver is shown in figure 10. In this sequence the $V h_{3}$ is far away, so the overtaking has been completed increasing the speed of $V h_{1}$. 

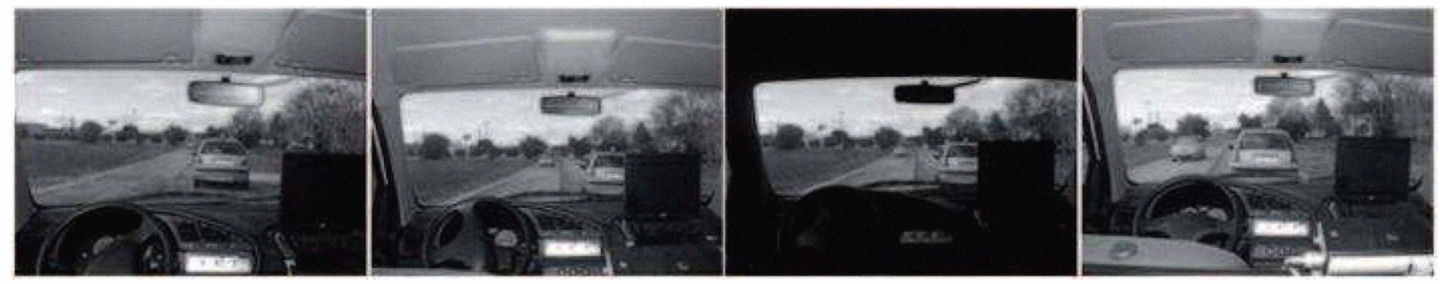

Fig. 9. Steps of an aborted overtaking in the experiment 1
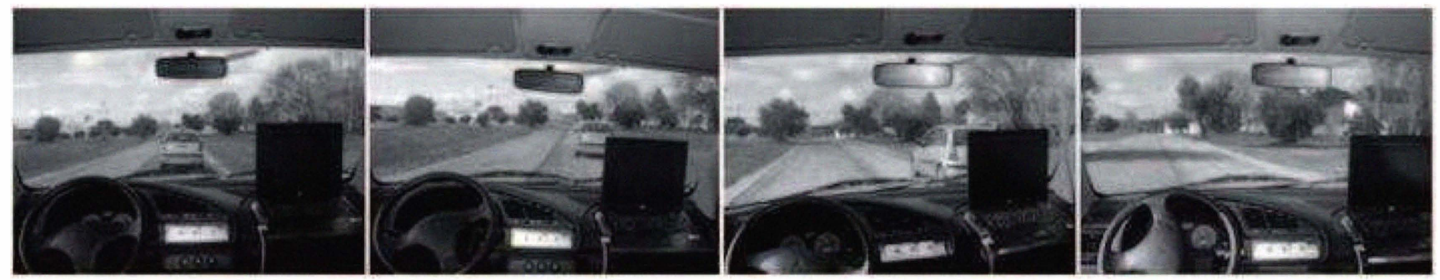

Fig. 10. Steps of an aborted overtaking in the experiment 2

\section{CONCLUSIONS}

In this paper, a new longitudinal architecture for the control of autonomous vehicles in highly risky situations is presented. It has been implemented and tested in real vehicles, using an automated electric van, which has been controlled through fuzzy rules, and two mass-produced Citroën C3.

Two autonomous overtaking modes have been tested; the normal and an extended overtaking. The former has used a target following control that maintains a predefined speed, in case of no risk exists. The latter uses the AFC to abort, if necessary, the overtaking maneuver.

Specifically, a novel architecture of the longitudinal fuzzy controller to take into consideration the possible cases in which the overtaking maneuver can be hindered by other vehicles is presented in this work.

Different tests to prove the system designed have been included. These experiments show the good behavior of the implemented AFC, even if the size of the test area does not permit high speeds.

In future work we will consider to increase the speed of the tests and to develop a lateral control that considered other environment variables.

\section{ACKNOWLEDGMENT}

This work was supported by the Spanish Ministry of Science and Innovation by means of Research Grant TRANSITO TRA2008-06602-C03 and Spanish Ministry of Development by means of Research Grant GUIADE P9/08.

\section{REFERENCES}

[1] C. D. Crane, "The 2005 darpa grand challenge," in Proc. Int. Symp. Computational Intelligence in Robotics and Automation CIRA 2007, 2007.

[2] "http://www.fomento.es/mfom/."

[3] J. Perez, V. Milanes, J. Alonso, E. Onieva, and T. de Pedro, “Overtaking with autonomous vehicles on two-way roads," Revista Iberoamericana de Automática e Informática Industrial, vol. 7, pp. 25-33, 2010.
[4] H. Jula, E. B. Kosmatopoulos, and P. A. Ioannou, "Collision avoidance analysis for lane changing and merging," vol. 49, no. 6, pp. 2295-2308, 2000.

[5] R. Rajamani, H.-S. Tan, B. K. Law, and W.-B. Zhang, "Demonstration of integrated longitudinal and lateral control for the operation of automated vehicles in platoons," vol. 8, no. 4, pp. 695-708, 2000.

[6] J. E. Naranjo, C. Gonzalez, T. de Pedro, R. Garcia, J. Alonso, M. A. Sotelo, and D. Fernandez, "Autopia architecture for automatic driving and maneuvering," in Proc. IEEE Intelligent Transportation Systems Conf. ITSC '06, 2006, pp. 1220-1225.

[7] D. C. K. Ngai and N. H. C. Yung, "Automated vehicle overtaking based on a multiple-goal reinforcement leaming framework," in Proc. IEEE Intelligent Transportation Systems Conf. ITSC 2007, 2007, pp. 818-823.

[8] S. Jamison and M. McCartney, "A vehicle overtaking model of traffic dynamics," American Institute of Physics, vol. 17, 2007.

[9] G. Usman and F. Kunwar, "Autonomous vehicle overtaking- an online solution," in Proc. IEEE Int. Conf. Automation and Logistics ICAL '09, 2009, pp. 596-601.

[10] G. Stanek, D. Langer, B. Muller-Bessler, and B. Huhnke, "Junior 3: A test platform for advanced driver assistance systems," in Proc. IEEE Intelligent Vehicles Symp. (IV), 2010, pp. 143-149.

[11] J. Alonso, J. Jiménez, J. E. Naranjo, J. Suárez, and B. Vinagre, "Cooperative maneuver study between autonomous cars: Overtaking," in Computer Aided Systems Theory Ü EUROCAST 2007, ser. Lecture Notes in Computer Science, S. B. . Heidelberg, Ed. Springer-Verlag, 2007, vol. 4739/2007, pp. 1151-1158.

[12] J. Perez, C. Gonzalez, V. Milanes, E. Onieva, J. Godoy, and T. de Pedro, "Modularity, adaptability and evolution in the autopia architecture for control of autonomous vehicles," in Proc. IEEE International Conference on Mechatronics ICM 2009, 14-17 April 2009, pp. 1-5.

[13] J. E. Naranjo, C. Gonzalez, R. Garcia, and T. de Pedro, "Lane-change fuzzy control in autonomous vehicles for the overtaking maneuver," IEEE Transactions on Intelligent Transportation Systems, vol. 9, no. 3, pp. $438-450$, Sept. 2008.

[14] L. A. Zadeh, "A new direction in fuzzy logic-toward a computational theory of perceptions," in Proc. NAFIPS Fuzzy Information Processing Society 18th Int. Conf. of the North American, 1999, pp. 1-4.

[15] V. Milanés, J. Pérez, E. Onieva, C. González, and T. de Pedro, "Lateral power controller for unmanned vehicles," Electrical Review, vol. 86, no. 1, pp. 207-211, Jan. 2010.

[16] R. García, T. de Pedro, J. Naranjo, J. Reviejo, and C. Gonzalez, "Frontal and lateral control for unmanned vehicles in urban tracks," IEEE Inteligent Vehicle Symposion IV'2002, Versailles, France., 2002.

[17] M. Sugeno and M. Nishida, "Fuzzy control of model car," Fuzzy Sets and Systems, vol. 16, pp. 103-113, 1985.

[18] J. Pérez, F. Seco, V. Milanés, A. Jiménez, J. Díaz, and T. de Pedro, "An rfid-based intelligent vehicle speed controller using active traffic signals," Sensors, vol. 10, no. 6, pp. 5872-5887, 2010. 